Aims To describe the ways in which parents recognise and make decisions about their child's symptoms following discharge home after congenital heart interventions in the first year of life and their experiences of seeking help.

Methods This was a qualitative study involving semi-structured interviews with parents. Twenty-one parents were recruited to the study. Parents all had a child who had congenital heart surgery in their first year of life between September 2009 and October 2013 at one of three UK cardiac centres; the children had either died or were readmitted as an emergency following initial discharge.

Results Some parents were unable to identify any early warning signs at all. Others described symptoms of deterioration including: changes in feeding and appearance, respiratory distress, and subtle behavioural changes that are not routinely highlighted to parents at discharge. Several barriers to accessing prompt medical assistance were identified including parents feeling that their concerns were not taken seriously, and long wait times and lack of protocols at A\&E.

Conclusions Our study highlights behavioural symptoms as being a potentially under-emphasised sign of deterioration and identifies a number of barriers to parents accessing support when they are concerned. It is important that parents are encouraged to seek advice at the earliest opportunity and that those health professionals at the front line have access to the information they need in order to respond in an appropriate and timely way. A role for home monitoring was also noted as potentially useful in identifying at risk children who appear clinically well.

Funding National Institute for Health Research Health Services and Delivery Research programme (Project No: 10/2002/29) and the Health Foundation.

\section{G166 RISK OF DEATH OR EMERGENCY READMISSION FOLLOWING DISCHARGE AFTER INFANT CARDIAC INTERVENTION FOR CONGENITAL HEART DISEASE}

${ }^{1} S$ Crowe, ${ }^{2} \mathrm{D}$ Ridout, ${ }^{2} \mathrm{R}$ Knowles, ${ }^{3} \mathrm{~J}$ Tregay, ${ }^{3} \mathrm{~J}$ Wray, ${ }^{4} \mathrm{D}$ Barron, ${ }^{5} \mathrm{D}$ Cunningham, ${ }^{6} \mathrm{R}$ Parslow, ${ }^{7} \mathrm{R}$ Franklin, ${ }^{3} \mathrm{C}$ Bull, ${ }^{3} \mathrm{~K}$ Brown. ${ }^{1}$ Clinical Operational Research Unit, University College London, London, UK; ${ }^{2}$ Institute of Child Health, University College London, London, UK; ${ }^{3}$ Great Ormond Street Hospital NHS Foundation Trust, London, UK; ${ }^{4}$ Birmingham Children's Hospital NHS Foundation Trust, Birmingham, UK; ${ }^{5}$ National Institute for Cardiovascular Outcomes Research, University College London, London, UK; ${ }^{6}$ Paediatric Intensive Care Audit Network, University of Leeds, Leeds, UK; ${ }^{7}$ Royal Brompton and Harefield Hospitals NHS Foundation Trust, London, UK

\subsection{6/archdischild-2015-308599.161}

Aims 1) To identify risk factors for death or unplanned readmission within one year following hospital discharge after cardiac intervention for congenital heart disease. 2) To characterise patient groups at highest risk who would benefit from targeted intervention.

Methods Records in the national congenital cardiac surgical audit (NICOR) pertaining to UK infants who had a cardiac surgery or intervention aged under 12 months between 01/01/2005 and 31/12/2010 were matched with intensive care admission records in Paediatric Intensive Care Audit Network (PICANET); linked records with known life-status were obtained for 7634 infants. Outcome measures were: Outcome 1 - death within 1-year following discharge; Outcome 2 - Outcome 1 or emergency readmission to PICU within 1-year following discharge. Potential risk factors available from either dataset were pre-specified and univariate and multivariate logistic regression used to investigate the effects of these on each outcome. Classification and regression tree (CART) analysis was used to identify distinct patient groups differentiated by risk of Outcome 2, each defined by a set of patient characteristics.

Results 3.2\% (246/7643) and 6.7\% (514/7643) of infants experienced Outcome 1 and 2 respectively. Fitted multivariate models for both outcomes were robust in risk factor selection (Outcome 1 - ROC AUC $=0.78,95 \%$ CI $[0.75,0.82]$; Outcome 2 - ROC $\mathrm{AUC}=0.78[0.75,0.80])$. Risk factors significant in the multivariate Outcome 2 model were: age at procedure, weight $\mathrm{z}$ score, cardiac procedure, cardiac diagnosis, non-cardiac congenital anomaly, neurodevelopmental condition, prematurity $(<37$ weeks gestation), ethnicity, and length of stay in specialist centre (LOS). Clinical deterioration was additionally significant to Outcome 1 whilst neurodevelopmental condition and acquired diagnoses were not. Key defining characteristics of infants in the patient groups identified as higher risk were [\% Outcome 2]: (1) neurodevelopmental conditions [24\%]; (2) Hypoplastic left heart, single ventricle or pulmonary atresia [15\%]; (3) Congenital anomalies and LOS > 1 month [24\% risk]; (4) No congenital anomalies and LOS $>1$ month [9\% risk].

Conclusions Understanding patient risk groups should inform recommendations for improving services, support development of interventions to mitigate each profile of risk and facilitate evaluation of the priority and feasibility of targeting each group.

\section{G167(P) CHEST PAIN IN CHILDREN IS RARELY CARDIAC. DOES ECG HELP?}

P Babu, S Dickson, D Dhar, D Hapuarachchi, K Murtagh, F Damda. Department of Paediatrics, University Hospital Lewisham, Lewisham and Greenwich NHS Trust, Lewisham, UK

\subsection{6/archdischild-2015-308599.162}

Aims Chest pain is a common reason for presentation to the children's emergency department (ED). It is known that chest pain in children, compared to adults, is much less likely to be caused by cardiovascular disease. Electrocardiographs (ECGs) are cheap, fast and readily available. When interpreted appropriately they can be useful in demonstrating cardiac causes of chest pain. We aimed firstly to determine the incidence and likely causes of paediatric chest pain presenting to our busy Children's ED and secondly to analyse the usefulness of ECGs in this cohort.

Methods We retrospectively analysed the ED case notes of all children (aged under 16 years) presenting with chest pain over a 4 year period (April 2009 to March 2013) to a busy Children's ED in an urban district general hospital.

Results 1126 attendances presented with chest pain, approximately $1 \%$ of all attendances over four years. Of those with chest pain 54\% were male and the modal age of presentation was 12 years. Based on history and clinical examination the commonest cause attributed to chest pain was musculoskeletal. Only $1 \%$ of cases had a possible cardiac aetiology; 1 patient had pericarditis, 1 patient had myocarditis, 2 patients had a pre-existing cardiac condition, 3 patients had arrhythmias and 8 were under investigation for recurrent palpitations. Thirty per cent of patients with chest pain had an ECG carried out. Patients with cardiovascular, psychiatric and musculoskeletal diagnoses were most likely to have had an ECG done. The majority of ECGs were normal (91\%). The commonest abnormality was high take off/mild ST elevation, with only $10 \%$ of such patients having cardiac enzymes requested. Some of the ECG abnormalities identified could not be attributed to chest pain. 
Conclusion Incidence of chest pain presenting to our ED was $1 \%$. The commonest recorded cause was musculoskeletal. Fewer than $1 \%$ had a possible cardiac aetiology for chest pain. ECG is a useful test for children presenting with chest pain. Very few patients with mild ST elevation had cardiac enzyme levels checked.

\section{G168(P) ANTENATAL MANAGEMENT OF FETAL CARDIAC DISEASES, A SINGLE CENTRE EXPERIENCE IN EGYPT}

H ElMarsafawy, S Rakha, R El Ashry, Y Al Tonbary. Department of Pediatrics, Faculty of Medicine, Mansoura University, Mansoura, Egypt

\subsection{6/archdischild-2015-308599.163}

Aims The antenatal diagnosis of congenital heart diseases (CHD) is crucial for fetal and perinatal management; however, there is little in literature regarding the pharmacologic intervention for fetal CHD in Egypt. Therefore, we attempted to detect fetal cardiac structural or functional abnormalities using fetal echocardiography and plan fetal pharmacologic intervention without endangering the mother's life.

Methods Cases fulfilled inclusion criteria were diagnosed using detailed fetal Echocardiographic examinations and antenatal treatment was described if indicated after a written consent. Cardiovascular profile score (CVPS) was used to assess the response to treatment. Postnatal transthoracic Echo was done to confirm diagnosis with follow up till the end of neonatal period to determine outcome.

Results Fetuses fulfilled inclusion criteria were 143 with a mean gestational age at diagnosis $27.59 \pm 5.41$ weeks, mean maternal age at diagnosis was $26.64 \pm 5.428$ years, the most frequent cause of referral was family history of CHD (34.1\%). Twenty fetuses (14\%) received antenatal therapy. Fetuses with heart failure due to structural cardiac defects $(n=4)$ and functional non arrhythmic heart failure $(n=8)$ received digoxin while cases with fetal tachyarrhythmia $(n=6)$ received digoxin and/or sotalol or flecainide and fetuses with immune mediated fetal heart block $(n=2)$ received dexamethazone. Success in tachyarrhythmia was statistically significant regarding CVPS $(p=0.038)$ and heart rate changes $(p=0.002)$ but statistically insignificant regarding CVPS in structural defects $(p=0.102)$ and nonarrhythmic functional heart failure $(p=0.343)$.

Conclusion Antenatal cardiac pharmacologic intervention is possible with hydrops fetalis reversal in fetal tachyarrhythmia and resolution of first degree immune mediated atrioventricular block. On the contrary, no response to antenatal digoxin use in fetal structural heart failure and limited response in non-arrhythmic functional heart failure.

\section{G169(P) EXPLORING ETHNIC VARIATION IN INFANTS WITH CONGENITAL HEART DEFECTS UNDERGOING PAEDIATRIC CARDIAC SURGERY}

\footnotetext{
${ }^{1} \mathrm{RL}$ Knowles, ${ }^{1} \mathrm{D}$ Ridout, ${ }^{2} \mathrm{~S}$ Crowe, ${ }^{3} \mathrm{~J}$ Tregay, ${ }^{3} \mathrm{~J}$ Wray, ${ }^{4} \mathrm{D}$ Barron, ${ }^{5} \mathrm{D}$ Cunningham, ${ }^{6} \mathrm{R}$ Parslow, ${ }^{7} \mathrm{R}$ Franklin, ${ }^{3} \mathrm{C}$ Bull, ${ }^{3} \mathrm{~K}$ Brown. ${ }^{1}$ Institute of Child Health, University College London, London, UK; ${ }^{2}$ Clinical Operational Research Unit, University College London, London, UK; ${ }^{3}$ Great Ormond Street Hospital NHS Foundation Trust, London, UK; ${ }^{4}$ Birmingham Children's Hospital NHS Foundation Trust, Birmingham, UK; ${ }^{5}$ National Institute for Cardiovascular Outcomes Research, University College London, London, UK; ${ }^{6}$ Paediatric Intensive Care Audit Network, University of Leeds, Leeds, UK; ${ }^{7}$ Royal Brompton and Harefield Hospitals NHS Foundation Trust, London, UK
}

Introduction North American researchers have reported ethnic differences in the prevalence and short-term outcomes of congenital heart defects (CHDs), which may reflect genetic variation, environmental exposures or healthcare access. It is unclear whether ethnic differences in CHD frequency and outcomes also exist in the UK population and healthcare system.

Aim To examine national paediatric cardiac surgical audit data for ethnic differences in the frequency of different CHD subtypes, associated comorbidities and short-term outcomes for infants operated in the first year of life.

Methods Individual records from the national congenital cardiac surgical audit (NICOR) of UK infants aged under 12 months who had a cardiac surgery or intervention between 01/01/2005 and 31/12/2010, were matched with intensive care admission records in the Paediatric Intensive Care Audit Network (PICANET) and linked records were obtained for 8481 (86\%) of operated infants. Census-derived categories for ethnic classification were used.

Results Children who were operated within the first year of life for major CHD represented 2.2 (95\% Confidence Intervals [CI] 2.2, 2.3) per 1000 live births. Compared with children of white ethnicity, children of Asian ethnicity were more likely to have cardiac surgery $(2.3[2.3,2.4]$ and $3.2[3.0,3.5]$ per 1000 live births respectively) in the first year of life. CHD subtypes that were significantly over-represented within the Asian ethnic group included single ventricle (SV), transposition of the great arteries, pulmonary atresia, tetralogy of Fallot (TOF) and septal defects; in the Black ethnic group, atrioventricular septal defect and SV were over-represented, while TOF and aortic stenosis were under-represented. Preterm birth occurred in 14\% of babies, almost twice the general population rate, and associated non-cardiac anomalies were reported in $21 \%$ of affected infants, however no significant ethnic variation was observed. There were 246 deaths during the first year after hospital discharge following surgery but no significant ethnic differences in short-term mortality were identified.

Conclusion The risk of CHD intervention in infants aged under one year varied by ethnic group, and children of Asian ethnicity were at greater risk. No ethnic differences in short-term post-discharge mortality were identified, however longer-term outcomes should be explored.

\section{G170(P) IMPROVING ANTENATAL DETECTION RATES OF SIGNIFICANT CONGENITAL CARDIAC LESIONS IN A DISTRICT GENERAL HOSPITAL}

H Aughey, L Guilder, Y Kumar. Paediatric Department, Royal Cornwall Hospital Trust, Truro, UK

\subsection{6/archdischild-2015-308599.165}

Introduction Congenital heart disease (CHD) affects around 1\% of pregnancies in the UK each year. Around half of these are major cardiac lesions requiring surgery or intervention within the $1^{\text {st }}$ year of life. Studies show that if CHD is detected before birth, there are significant benefits for babies, their families and for medical services around the time of birth and in the first year of life. Prenatal diagnosis and appropriate treatment may prevent the devastating consequences of early circulatory collapse, such as death and ischaemic brain damage.

Background An audit previously conducted in our district general hospital found that the antenatal detection rate of significant cardiac lesions in Jan 1998-Dec 1999 was 17.6\%. Following 